\title{
Polarization Insensitivity of AlGaAs/GaAs Interdiffused Quantum Wells
}

\author{
Wallace C. H. Choy ${ }^{a}$, E. Herbert $\mathrm{Li}^{\mathrm{b}}$, and Bernard L. Weiss ${ }^{\mathrm{a}}$ \\ ${ }^{a}$ School of Electronic Engineering, Information Technology and Mathematics, University of Surrey, Guildford, Surrey, \\ GU2 5XH, UK \\ ${ }^{\mathrm{b}}$ Department of Electrical \& Electronic Engineering, University of Hong Kong, \\ Pokfulam Road, Hong Kong
}

\begin{abstract}
Modeling is used to show that interdiffusion can generate a polarization independent parabolic-like quantum well. Criteria to achieve the parabolic-like quantum wells by interdiffusion are discussed. The results indicated that interdiffused quantum wells can produce equal eigen-state spacing, polarization insensitive Stark shift and modulation characteristics similar to an ideal parabolic quantum well. The design process to obtain polarization insensitive ON- and OFF- states in the parabolic-like interdiffused quantum wells is discussed. The predicted modulation depth is comparable to those measured using parabolic quantum wells. The diffused quantum wells have the advantage of using an as-grown rectangular quantum well with post-growth annealing to tailor its confinement profile. These features suggest that the interdiffused quantum well structure can be use to produce polarization insensitive electro-absorptive modulation.
\end{abstract}

\section{INTRODUCTION}

Recently, polarization insensitive electro-absorptive III-V semiconductor quantum well (QW) modulation devices have been under intensive development because the polarization of an incoming optical signal is random [1]. One of the ways to produce the polarization insensitivity is to develop a parabolic-shaped confinement in the finite depth QW structure (hereafter denote as PQW) [2]. The reason for this is that the quantum confined Stark shift of the fundamental transitions are insensitive to the heavy hole $(\mathrm{HH})$ and light hole $(\mathrm{LH})$ effective masses in the PQW, thereby resulting in an identical energy of the Stark shift [3]. This feature gives the PQW an ability to modulate light with a transverse magnetic (TM) polarization in a similar degree to that with a transverse electric (TE) polarization. However, it is difficult to fabricate a PQW accurately, in particular, to produce multiple numbers of these PQWs with good uniformity. In this paper, using the simple technology of thermal annealing of the QW [4], three interdiffusion induced parabolic-like $\mathrm{Al}_{\mathrm{x}} \mathrm{Ga}_{1-\mathrm{x}} \mathrm{As} / \mathrm{GaAs} \mathrm{QW}$ structures with $\mathrm{Al}$ concentration of $\mathrm{x}=0.4,0.35$ and 0.3 are generated for the polarization independence. The criteria to achieve the interdiffused QW (DFQW) and the steps to obtain polarization insensitive ON-state and OFF-state are discussed. The polarization insensitive modulation properties of the optimized DFQW structures are then addressed.

\section{MODELING}

The three PQW structures we aim to achieve are: firstly one with a high Al concentration $(x)$ of 0.4 and a well width $\left(L_{z}\right)$ of $22 \mathrm{~nm}$ [5], secondly one with $\mathrm{x}=$ of 0.35 and $\mathrm{L}_{\mathrm{z}}=21 \mathrm{~nm}$, and thirdly one with $\mathrm{x}=0.3$ and $\mathrm{L}_{\mathrm{z}}=20 \mathrm{~nm}$ [3]. The corresponding parabolic DFQWs with polarization insensitive $\mathrm{ON}$ and OFF states are: firstly one with $\mathrm{x}=0.37, \mathrm{~L}_{\mathrm{z}}=17 \mathrm{~nm}$ and $\mathrm{L}_{\mathrm{d}}=2.2 \mathrm{~nm}$ (denoted as DFQW(hi-Al) hereafter), secondly one with $\mathrm{x}=0.35, \mathrm{~L}_{\mathrm{z}}=16 \mathrm{~nm}$ and $\mathrm{L}_{\mathrm{d}}=2.1 \mathrm{~nm}$ (denoted as $\mathrm{DFQW}\left(\right.$ mid-Al)), and lastly one $\mathrm{x}=0.32, \mathrm{~L}_{z}=14 \mathrm{~nm}$ and $\mathrm{L}_{d}=2 \mathrm{~nm}$ (denoted as DFQW(low-Al)), where $\mathrm{L}_{z}$ is the as-grown well width of the DFQW. The extent of the interdiffusion process in the DFQW is characterized by a diffusion length $\mathrm{L}_{\mathrm{d}}$, which is defined by $L_{d}=(D t)^{1 / 2}$, where $\mathrm{D}$ and $t$ are the diffusion coefficient and the annealing time respectively [6]. The QW subband structure is modeled by solving a one-dimensional one particle Schrödinger-like equation with an effective mass approximation [7]. The absorption coefficient $(\alpha)$ is calculated using a density matrix approach [8]. Two features of the PQW are used to determine the parabolicity of the DFQW potential profile of the DFQW. They are (i) the spacing of adjacent eigenstates, i.e. the energy difference of two adjacent eigenstates $\Delta E_{i j}$ where the subscripts $i$ and $j$ are the states number is identical to that of any other two adjacent PQW confined eigenstates, and (ii) an equal Stark shift energy of the transitions between the first electron and first heavy hole state $(\mathrm{C} 1-\mathrm{HH} 1)$ and between the first electron and first light hole 
state (C1-LH1) as well as the shift of their 1S exciton transition energies. The performance of the modulator is discussed in terms of the quantum confined Stark shift (QCSS), the absorption change $(\Delta \alpha)$, the absorption loss $\left(\alpha_{\text {loss }}\right)$, the contrast ratio (CR) and the operating wavelength $\left(\lambda_{\text {op }}\right)$.

\section{RESULTS AND DISCUSSIONS}

\subsection{Criteria for parabolic DFQW}

With regard to the first criterion, a narrow as-grown well width is required because the width of the upper half of the DFQW potential profile increases (dotted-dash line) as $L_{d}$ increases, as shown in Fig. 1. With an appropriate $L_{d}$, i.e. $2 \mathrm{~nm}$ (solid line), the diffused profile becomes very close to that of a PQW (dash line). The second criterion is to minimize the mean deviation of all $\Delta \mathrm{E}_{\mathrm{ij}}$ taking into account all the confined states. Due to interdiffusion, the bound states will change and thus $\Delta \mathrm{E}_{\mathrm{ij}}$ is modified, as shown in Table 1. The deviation of $\Delta \mathrm{E}_{\mathrm{ij}}$ for all transitions between the conduction band and the $\mathrm{HH}$ valence band, i.e $\Delta \mathrm{E}_{12}$ and $\Delta \mathrm{E}_{54}$, reduces as $\mathrm{L}_{\mathrm{d}}$ increases as the confinement profile becomes more parabolic-like in shape. The mean deviation of $\Delta \mathrm{E}_{\mathrm{ij}}$ reduces as $\mathrm{L}_{\mathrm{d}}$ increases up to a certain value of $\mathrm{L}_{\mathrm{d}}$, as shown in Fig. 2,. The mean deviation of $\Delta \mathrm{E}_{\mathrm{ij}}$ becomes a minimum at $\mathrm{L}_{\mathrm{d}}=2 \mathrm{~nm}$ and $\mathrm{L}_{\mathrm{d}} \approx 1.5 \mathrm{~nm}$ for the $\mathrm{HH}$ and electron eigenstates respectively, see Fig. 2. Further increases of $\mathrm{L}_{d}$ the DFQW confinement profile becomes less parabolic-like so that the mean deviation increases. The last criterion is to minimize the difference of C1-HH1 and C1-LH1 QCSS. The minimum is obtained at $\mathrm{L}_{d}=2 \mathrm{~nm}$. The difference of the minimum of the mean deviation of $\mathrm{HH}$ bound state and the minimum of the QCSS indicates that the DFQW with $L_{d}=2 n m$ is nearest to a parabolic-like shape for the $Q W$ structure with $(x)=0.32$ and $L_{z}=14 n m$. Consequently, this DFQW structure is almost polarization insensitivity. Similarly, DFQW structures with the following parameters (i) $\mathrm{x}=0.35, \mathrm{~L}_{\mathrm{z}}=16 \mathrm{~nm}$ and $\mathrm{L}_{\mathrm{d}}=2.1 \mathrm{~nm}$, and (ii) $\mathrm{x}=0.37, \mathrm{~L}_{\mathrm{z}}=17 \mathrm{~nm}$ and $\mathrm{L}_{\mathrm{d}}=2.2 \mathrm{~nm}$ are polarization insensitive.

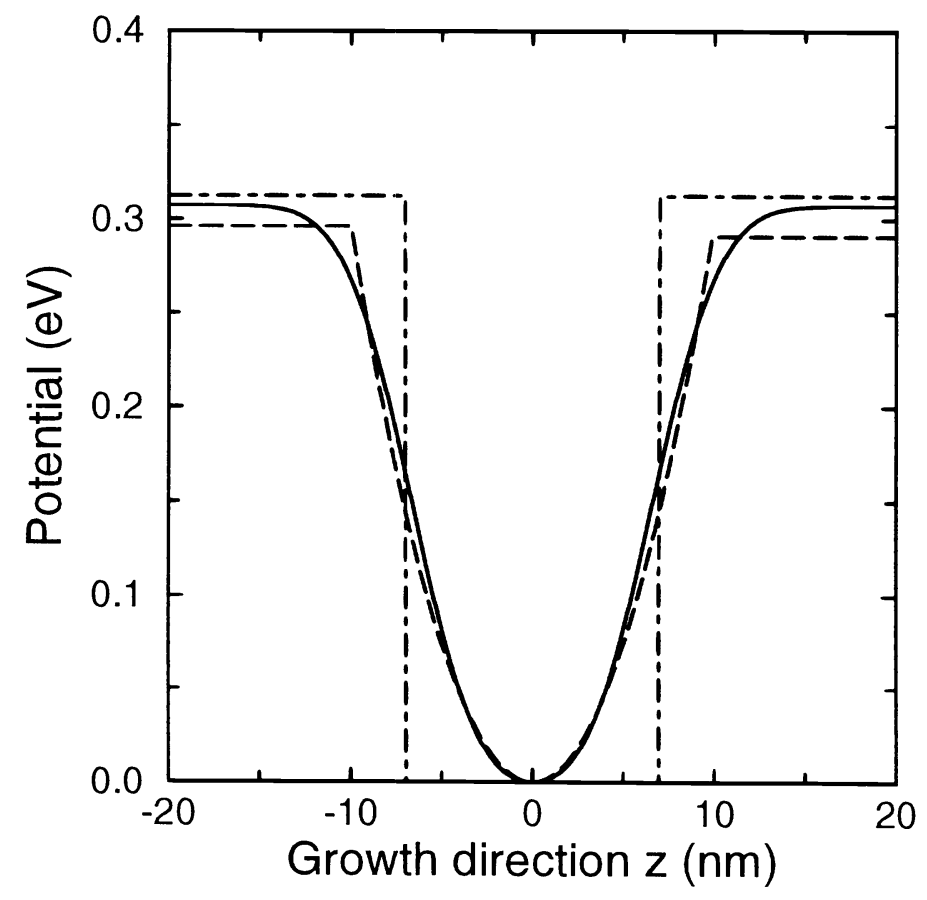

Fig. 1 Potential profile of the parabolic DFQW (solid line) with $x=0.32, L_{z}=14 n m, L_{d}=2.0 \mathrm{~nm}$, and its corresponding as-grown QW (dashed-dot line) with $\mathrm{L}_{\mathrm{d}}=0$, and the targeted PQW (dash line) $\mathrm{x}=0.3, \mathrm{~L}_{\mathrm{z}}=20 \mathrm{~nm}$. 


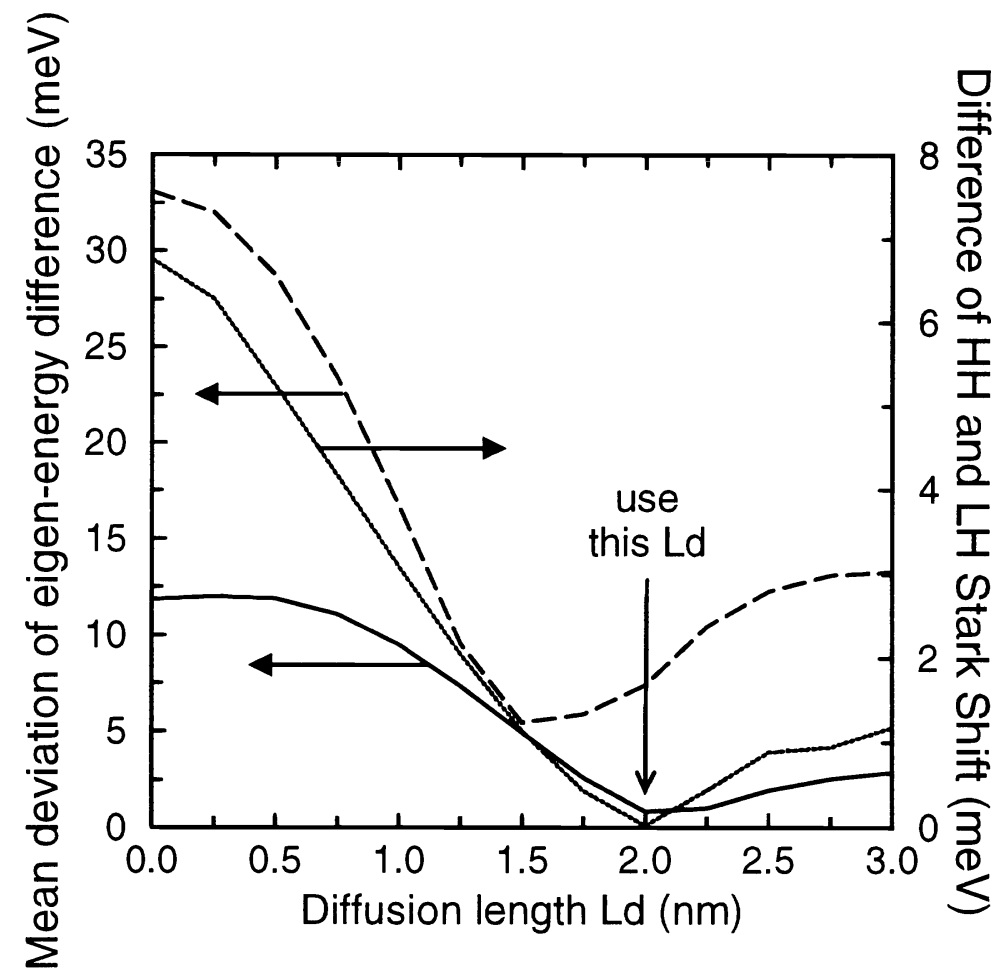

Fig. 2 Mean deviation of $\Delta \mathrm{E}_{\mathrm{ij}}$ of $\mathrm{HH}$ (solid line) and electron (long dash line), and difference between C1-HH and C1-LH QCSS (dot line) of the DFQW with $\mathrm{x}=0.32$.

Table 1 Energy difference between adjacent bound states: $i$ and $j, \Delta E_{i j}$, in conduction band and $\mathrm{HH}$ valence band of DFQW with aluminum content $\mathrm{x}=0.32$ and $\mathrm{L}_{\mathrm{z}}=14 \mathrm{~nm}$.

\begin{tabular}{lllll}
\hline $\mathrm{L}_{\mathrm{d}}(\mathrm{nm})$ & $\Delta \mathrm{E}_{21}(\mathrm{meV})$ & $\Delta \mathrm{E}_{32}(\mathrm{meV})$ & $\Delta \mathrm{E}_{43}(\mathrm{meV})$ & $\Delta \mathrm{E}_{54}(\mathrm{meV})$ \\
\hline \multicolumn{5}{c}{ heavy hole } \\
\hline 0 & 9.3 & 15.3 & 21.2 & 26.8 \\
0.5 & 10.5 & 17.1 & 22.9 & 27.4 \\
1 & 13.5 & 20 & 23.8 & 25.3 \\
1.5 & 17.3 & 21.4 & 22.8 & 22.4 \\
2 & 20.1 & 21.5 & 22 & 19.8 \\
2.5 & 20.8 & 20.2 & 19 & 17.4 \\
3 & 19.9 & 18.7 & 17.2 & 15.5 \\
\hline \multicolumn{5}{c}{ electron } \\
\hline 0 & 59.1 & 96.5 & 120.9 & \\
0.5 & 63.9 & 100.2 & 113.7 & \\
1 & 73 & 101.2 & 95 & \\
1.5 & 82.1 & 94.4 & 78.1 & \\
1.75 & 85.1 & 89.5 & 71.8 & \\
2 & 86.5 & 84.4 & 66.6 & \\
2.5 & 84.9 & 74.9 & 58.2 & \\
3 & 79 & 66.6 & 54.7 & \\
\hline
\end{tabular}




\subsection{Polarization insensitive ON/OFF electro-absorption modulation}

Polarization insensitive ON-state is an unbiased state in which $\alpha_{\mathrm{TE}}=\alpha_{\mathrm{TM}}$ and the polarization OFF-state is a bias state in which $\alpha_{\mathrm{TE}}=\alpha_{\mathrm{TM}}$. In order to obtain these $\mathrm{ON}$ and $\mathrm{OFF}$ states with a polarization insensitive electro-absorptive change (i.e. $\Delta \alpha_{\mathrm{TE}}=\Delta \alpha_{\mathrm{TM}}$ ), the operating wavelength and the strength of the reverse biased field are two crucial factors that have to be determined. The results indicate that the operating wavelength and the biased field are inter-related. The determination of the ON- and OFF- states by interdiffusion are discussed followed by the electro-absorption modulation of the various QW structures.

Initially the range of $\lambda_{\text {op }}$ within which the modulator can provide polarization insensitive ON-state is determined. This range can be determined from the unbiased $(F=0)$ TE and TM absorption spectra, as shown in Fig 3 for a DFQW with $\mathrm{x}=0.32$. Due to the broadening of exciton absorption, the same TE and TM absorption coefficient can only be found in the absorption tail at least $10 \mathrm{~nm}$ away from the exciton edges (HH for TE and LH for TM). The wavelength of the absorption tail with same TE and TM absorption coefficient starts at $\sim 0.858 \mu \mathrm{m}$ up to $0.88 \mu \mathrm{m}$ which is the $\lambda_{\text {op }}$ range over which a polarization insensitive $\mathrm{ON}$ state can be obtained.

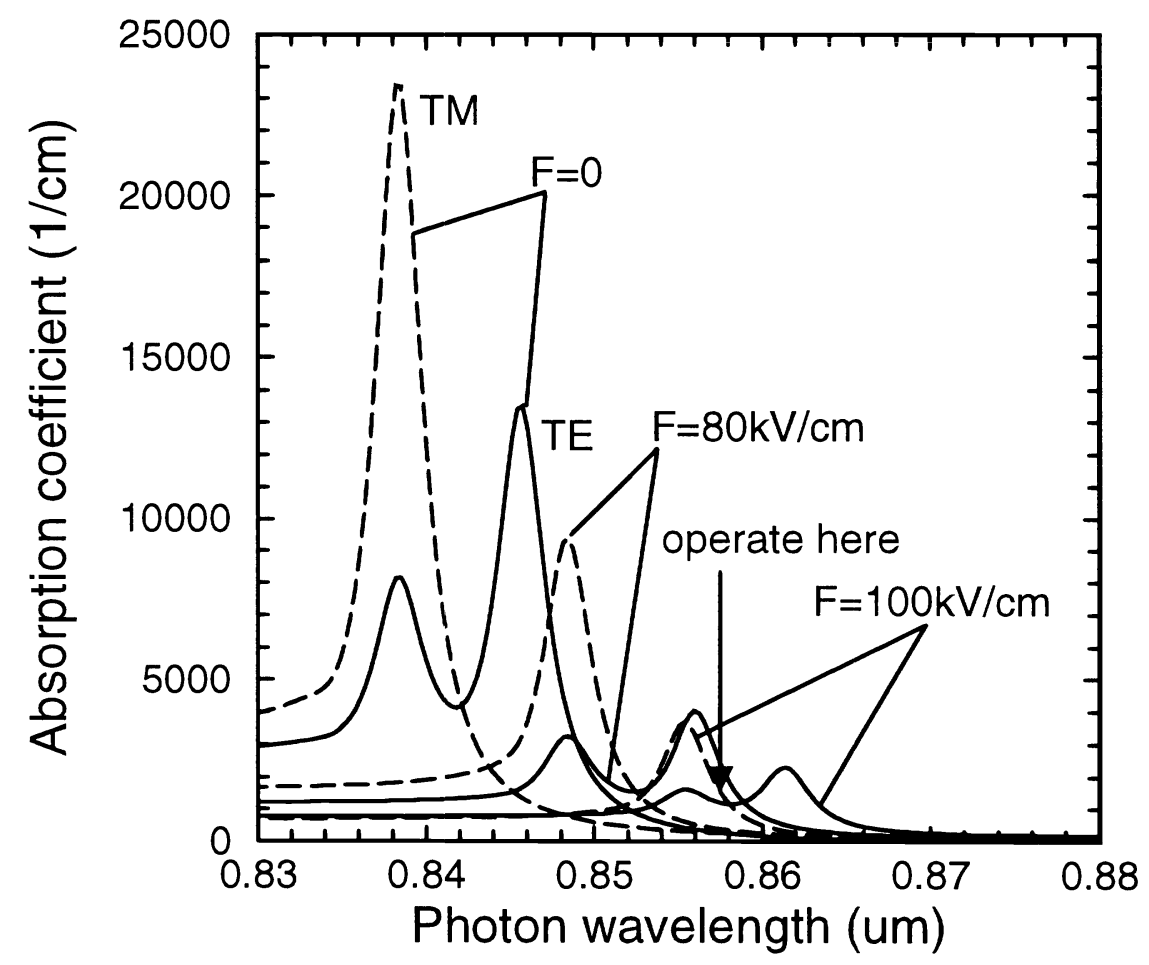

Fig. 3 The absorption spectra of the TE and TM polarization modes of the DFQW with low aluminum concentration, $\mathrm{x}=0.32$ (low-Al).

Subsequently, the range of bias fields with which produce a polarization insensitive OFF-state can operate within the predetermined range of wavelengths with same amount of unbiased TE and TM absorption (obtained from step 1) is determined. For the case shown in Fig. 3, when $\mathrm{F} \geq 100 \mathrm{kV} / \mathrm{cm}$, the cross point of biased TE and TM absorption (the polarization insensitive OFF-state) can shift due to Stark effect into the wavelength range. The wavelength of this cross point is the $\lambda_{\text {op }}$ with polarization insensitive ON- and OFF- states. Since an applied field $F \geq 100 \mathrm{kV} / \mathrm{cm}$ can be used to produce the ON- and OFF- states, more than one $\lambda_{\text {op }}$ can be obtained for polarization insensitive modulation. 
In the final step, the most appropriate $\lambda_{\text {op }}$ and bias field are determined. When the applied field increases, the absorption coefficient reduces because the overlap integral of both the electron and heavy hole wavefunctions and the electron and light hole wavefunction are reduced. In the case of Fig. 3, when the applied field increases above $\mathrm{F}=100 \mathrm{kV} / \mathrm{cm}$, polarization insensitive ON- and OFF- states can be obtained, although the absorption coefficient of the OFF-state and $\Delta \alpha$ (absorption change between the OFF-state and the ON-state) are reduced. In view of this, we have to consider the trade-off between $\alpha_{\text {loss }}\left(\alpha\right.$ at the OFF-state ) and $\Delta \alpha$. When an applied field increases, $\alpha_{\text {loss }}$ reduces since $\lambda_{\text {op }}$ moves far from the unbiased exciton edges (high loss), although $\Delta \alpha$ (and thus CR) are also reduced. In order to have a large $\Delta \alpha$ and, at the same time, to maintain polarization insensitive $\mathrm{ON}$ - and OFF- states, $\mathrm{F}=100 \mathrm{kV} / \mathrm{cm}$ should be used as the bias field and $\lambda_{\text {op }}$ is now set at $0.858 \mu \mathrm{m}$ for the case of DFQW(low-Al), as shown in Fig. 3. Since $\lambda_{\mathrm{op}}$ is far from the unbiased HH exciton absorption edge $(0.846 \mu \mathrm{m}), \alpha_{10 s s}$ is low with value of $258 \mathrm{~cm}^{-1}$. It should be noted that, since $\lambda_{\text {op }}$ increases with applied field, these two parameters are inter-related.

The applied field and $\lambda_{\text {op }}$ for the other two cases DFQW(mid-Al) and DFQW(hi-Al) may also determined Using a similar method for both of these DFQWs with a field of $100 \mathrm{kV} / \mathrm{cm}$ and their $\lambda_{\text {op }}$ set at $0.861 \mu \mathrm{m}$ and $0.863 \mu \mathrm{m}$ respectively. The modulation properties of all the three cases, including the ON-state and OFF-state polarization insensitive absorption coefficient and CR with a modulation interaction length of $50 \mu \mathrm{m}$ are given in Table $2 . \alpha_{\text {loss }}$ reduces from $258 \mathrm{~cm}^{-1}$ to $200 \mathrm{~cm}^{-1}$ while the $\mathrm{CR}$ is also reduced from $20 \mathrm{~dB}$ to $13 \mathrm{~dB}$, by comparing the DFQW(low-Al) with the DFQW(hi-Al). This is mainly because the well width of the DFQW increases from $14 \mathrm{~nm}$ to $17 \mathrm{~nm}$, so that $\alpha$ reduces for $\mathrm{x}=0.32$, as shown in Fig. 3. Comparing the results of this DFQW(low-Al) with PQW measurements [3] gives $\Delta \alpha$ of $\sim 1000 \mathrm{~cm}^{-1}$ for the PQW against $1162 \mathrm{~cm}^{-1}$ for the DFQW for $\lambda_{\text {op }}$ of $0.858 \mu \mathrm{m}$. This implies that the DFQWs can perform as well as the PQWs.

Table 2 The modulation properties of the three parabolic-like DFQWs polarization insensitive modulators.

\begin{tabular}{ccccc}
\hline case & $\lambda_{\mathrm{op}}(\mu \mathrm{m})$ & ON-state $\alpha\left(\mathrm{cm}^{-1}\right)$ & $\Delta \alpha\left(\mathrm{cm}^{-1}\right)$ & $\mathrm{CR}(\mathrm{dB})$ \\
\hline low-Al & 0.858 & 258 & 1162 & 20 \\
mid-Al & 0.861 & 240 & 1109 & 19 \\
hi-Al & 0.863 & 200 & 816 & 13 \\
\hline
\end{tabular}

It is known that, within realistic experimental limits, a deviation of few monolayers is possible in QW layer thickness'. For example, a QW(low-Al) with a deviation of $\pm 5 \AA$ in well width, the polarization insensitive $\Delta \alpha$ will degrade from $1162 \mathrm{~cm}^{-1}$ $\left(\mathrm{L}_{\mathrm{z}}=14 \mathrm{~nm}, \lambda_{\text {op }}=0.858 \mu \mathrm{m}\right)$, as shown in Table 2 , to $971 \mathrm{~cm}^{-1}$ for $\mathrm{L}_{\mathrm{z}}=13.5 \mathrm{~nm}$ and to $715 \mathrm{~cm}^{-1}$ for $\mathrm{L}_{\mathrm{z}}=1.5 \mathrm{~nm}$. The $\Delta \alpha$ of the narrower QW is greater because the absorption coefficient is inversely proportional to $\mathrm{L}_{z}$. In addition, in the cases of $\mathrm{L}_{z}=$ $13.5 \mathrm{~nm}$ and $14.5 \mathrm{~nm}, \lambda_{\text {op }}$ blue shifts to $0.853 \mu \mathrm{m}$ and red shifts to $0.862 \mu \mathrm{m}$, respectively. In case of $\mathrm{L}_{z}=14.5 \mathrm{~nm}$, the optimized $\mathrm{L}_{\mathrm{d}}$ is $2.25 \mathrm{~nm}$. However, since both $\mathrm{L}_{\mathrm{z}}$ and $\mathrm{L}_{\mathrm{d}}$ are increase in comparison to that of the optimized structure for $\mathrm{L}_{\mathrm{z}}=14 \mathrm{~nm}$ with $\mathrm{L}_{\mathrm{d}}=2 \mathrm{~nm}$, the quantum confinement and thus $\Delta \alpha$ of the optimized $\mathrm{L}_{\mathrm{z}}=14.5 \mathrm{~nm}$ case decreases to $645 \mathrm{~cm}^{-1}$ [9]. Consequently, in order to develop a parabolic-like DFQW with larger absorption modulation, the as-grown well width, $\mathrm{Al}$ concentration in the barrier and the extent of the interdiffusion have to be selected carefully. The criteria discussed in section 2 above and the results analyzed in this section serve as a guideline to produce useful polarization insensitive absorption modulation.

\section{CONCLUSIONS}

The use of interdiffusion in different as-grown square QW structures to produce a parabolic-like potential profile which have polarization insensitivity of electro-absorptive modulation properties have been investigated theoretically. Three criteria to produce parabolic-like DFQW have been established. The procedures required to obtain polarization insensitive $\mathrm{ON}$ - and OFF- states electro-absorptive modulation are also proposed. The optimized results show that electro-absorptive modulation of the DFQW is as good as that of the as-grown PQWs reported previously [3]. As a consequence, the DFQWs can be used to produce polarization insensitive electro-absorption. 


\section{ACKNOWLEDGMENTS}

This work is supported by a grant from the Hong Kong Research Grants Committee. W.C.H. Choy acknowledges the award of a research scholarship of the Croucher Foundation. The authors would like to acknowledge the technical support of the Center for Vision, speech, and signal processing (CVSSP) of University of Surrey. E. H. Li is currently a visiting scholar at the Division of Engineering and Applied Sciences, Harvard University, Cambridge, MA 02138.

\section{REFERENCES}

1. T. Aizawa, Y. Nagasawa, K. G. Ravikumar and T. Watanabe, "Polarization-independent switching operation in directional coupler using tensile-strained multi-quantum well", IEEE Photon. Technol., vol. 7, pp.47-49, 1995.

2. R. C. Miller, A. C. Gossard, D. A. Kleinman and O. Munteamu, "Parabolic quantum wells with the GaAs-Al $\mathrm{Ga}_{1-\mathrm{x}} \mathrm{As}$ system", Phys. Rev. B, vol. 29, pp.3740-3743, 1984.

3. T. Ishikawa, S. Nishimura and K. Tada, "Quantum-confined Stark effect in a parabolic-potential quantum well", Jpn. J. of Appl. Phys., vol. 29, pp. 1466-1473, 1990.

4. E. H. Li, Ed., "Semiconductor quantum well intermixing", (Gordon \& Breach, Amsterdam, 1998).

5. Y. Huang and C. Lien, "The enhancement of optical third harmonic susceptibility in parabolic quantum well by triple resonance", J. Appl. Phys., vol. 75, pp. 3223-3225, 1994.

6. K. Kash, B. Tell, P. Grabbe, E. A. Dobisz, H. G. Graighead and C. M. Tamargo, "Aluminum ion-implantation enhanced intermixing of GaAs-AlGaAs quantum-well structures", J. Appl. Phys., vol. 63, pp.90-194, 1988.

7. W. C. H. Choy, E. H. Li and B. L. Weiss, "AlGaAs/GaAs quantum well electro-optic and electro-absorptive modulators using surface acoustic wave”, J. Appl. Phys., vol. 83, pp.868-866, 1998.

8. W. C. H. Choy, E. H. Li and J. Micallef, "The polarization insensitive Electro-absorptive and -refractive Modulation by Utilizing InGaAsP/InP interdiffused quantum well”, IEEE J. of Quantum Electron., vol. 33, pp.1316-1322, 1997.

9. B.Pezeshki, S.M. Lord, T.B. Boykin and J.S.Harris, Jr, "GaAs/AlAs quantum wells for electro-absorption modulators", Appl. Phys. Lett., vol. 60, pp 2779-2781, 1992. 